\title{
MicroRNA-382 inhibits cell proliferation and invasion of retinoblastoma by targeting BDNF-mediated PI3K/AKT signalling pathway
}

\author{
DAN SONG ${ }^{1}$, JIANDONG DIAO ${ }^{2}$, YONGJING YANG ${ }^{3}$ and YAHONG CHEN $^{4}$
}

\begin{abstract}
${ }^{1}$ Department of Ophthalmology, Beijing Tsinghua Changgung Hospital, School of Clinical Medicine, Tsinghua University, Beijing 102218; ${ }^{2}$ Department of Oncology and Hematology, China-Japan Union Hospital of Jilin University, Changchun, Jilin 130033; ${ }^{3}$ Department of Thoracic Oncology, Jilin Cancer Hospital, Changchun, Jilin 130012; ${ }^{4}$ Department of Colorectal Surgery, China-Japan Union Hospital of Jilin University, Changchun, Jilin 130033, P.R. China
\end{abstract}

Received March 6, 2017; Accepted August 16, 2017

DOI: $10.3892 / \mathrm{mmr} .2017 .7396$

\begin{abstract}
It has previously been demonstrated that multiple microRNAs (miRNAs or miRs) are aberrantly expressed in retinoblastoma (RB) and contribute to RB initiation and progression. miR-382 has been revealed to be aberrantly expressed and therefore exhibits a key role in the progression of various types of cancer. However, the expression pattern, functional roles and underlying molecular mechanism of miR-382 in $\mathrm{RB}$ remain unknown. The present study investigated the expression levels of miR-382 and its effects on RB cells and the underlying regulatory mechanism of its action. It was demonstrated that miR-382 was downregulated in RB tissues and cell lines. Upregulation of miR-382 inhibited RB cell proliferation and invasion in vitro. Additionally, brain-derived neurotrophic factor (BDNF) was identified as a novel target of miR-382 in RB. BDNF was upregulated in RB tissues and negatively associated with miR-382 expression levels. Furthermore, BDNF overexpression rescued the tumour-suppressing effects on RB cells induced by miR-382. miR-382 inactivated the phosphoinositide 3-kinase/protein kinase B (PI3K/AKT) signalling pathway in RB. These findings suggested that miR-382 serves as a tumour suppressor in $\mathrm{RB}$, in part, by targeting the BDNF-mediated PI3K/AKT signalling pathway. The results of the present study suggest a potential therapeutic strategy for treating RB patients in the future.
\end{abstract}

\section{Introduction}

Retinoblastoma (RB) is the most common intraocular malignant tumour that normally affects infants and young children,

Correspondence to: Professor Yahong Chen, Department of Colorectal Surgery, China-Japan Union Hospital of Jilin University, 126 Xiantai Road, Changchun, Jilin 130033, P.R. China

E-mail: chen_yahong943@163.com

Key words: retinoblastoma, microRNA-382, brain-derived neurotrophic factor, PI3K/AKT with a prevalence ranging from $1: 15,000$ to $1: 20,000$ in children under the age of 5 years in the USA $(1,2)$. RB can be classified into two groups: Hereditary and nonhereditary. Hereditary RB patients account for $30-40 \%$ cases and is usually associated with a positive family history; those patients with no family history are generally classified as nonhereditary (3-5). Previous studies demonstrated that high oncogene expression level, loss of tumour suppressors and epigenetic changes of oncogenic methylation may be involved in RB formation and progression (6-8). Currently, the primary treatments for RB are enucleation, chemotherapy and focal therapy, such as laser or cryotherapy (9). Despite improvements in therapeutic treatments, the prognosis for patients with RB remains poor, with a mortality rate of 50-70\% among children in underdeveloped countries (10). This poor outcome is largely due to diagnosis and treatment delay (11). Therefore, further exploration of the biology and molecular mechanisms of RB that cause RB procession and metastasis and investigation of novel therapeutic methods to improve the prognosis of patients with $\mathrm{RB}$ are necessary.

MicroRNAs (miRNAs or miRs) are a group of endogenous, single-strand, non-coding 19-25 nucleotide RNAs that modulate more than half of the genes in human cells (12). Over 1,000 miRNAs have been identified within the human genome (13), and they may regulate gene expression by interacting with complementary sites in the 3'-untranslated regions (3'-UTRs) of their target mRNA molecules, thereby leading to mRNA degradation or post-transcriptional translational repression (14). miRNAs act as key regulators in a wide variety of physiological and pathological processes, such as cell proliferation, cycle, differentiation, apoptosis, metabolism and death (15-17). An increasing number of studies reported miRNA dysregulation in various kinds of human cancers, such as gastric cancer (18), glioma (19), lung cancer (20), bladder cancer (21) and RB (22). The abnormally expressed miRNAs are involved in progression and development of various cancers and serve as oncogenic and tumour suppressors by directly targeting the oncogenes and tumour-suppressor genes, respectively (23-25). Several miRNAs were correlated with RB tumourigenesis and development and might serve as 
independent prognostic markers or therapy targets for patients with this malignancy (26-28). Therefore, aberrant miRNAs and their functions in RB need to be explored to benefit the discovery of novel diagnosis biomarkers and therapeutic strategies.

miR-382 is aberrantly expressed and play important roles in several types of cancer (29-31). However, the expression pattern, functional roles and underlying molecular mechanism of miR-382 in RB remains unknown. This study aimed to investigate the miR-382 expression levels in RB tissues and cell lines. Furthermore, the effects of miR-382 on RB cells and the underlying regulatory mechanism of its action were also examined.

\section{Materials and methods}

Tissue samples. This study was approved by the Human Subjects Committee of Beijing Tsinghua Chang Gung Hospital. Informed consent was also obtained from all patients. A total of $26 \mathrm{RB}$ tissues were obtained from patients who underwent enucleation at Beijing Tsinghua Chang Gung Hospital (Beijing, China). Nine normal retina samples were collected from paediatric ruptured globe. RB tissues were excluded from experiment if the RB patients had been treated with chemotherapy or other treatments prior to enucleation. None of the patients received thermotherapy, cryotherapy, chemotherapy and radiotherapy before surgery. Tissues were immediately snap frozen in liquid nitrogen and transferred at $-80^{\circ} \mathrm{C}$ in the refrigerator for storage.

Cell lines, culture conditions and transfection. Three human RB cell lines (Y79, WERI-RB-1 and SO-RB50) were purchased from the American Type Culture Collection (Manassas, VA, USA) and cultured in RPMI-1640 medium (Gibco, Grand Island, NY, USA) containing 10\% heat-inactivated fetal bovine serum (FBS; Gibco), $100 \mathrm{U} / \mathrm{ml}$ penicillin and $100 \mathrm{mg} / \mathrm{ml}$ streptomycin. All cells were grown in a humidified atmosphere at $37^{\circ} \mathrm{C}$ with $5 \% \mathrm{CO}_{2}$.

miR-382 mimics and miRNA mimic negative control (miR-NC) were obtained from Shanghai GenePharma Co., Ltd. (Shanghai, China). Brain-derived neurotrophic factor (BDNF) overexpression vector (pCDNA3.1-BDNF) and corresponding blank vector (pCDNA3.1) were synthesised by Guangzhou RiboBio Co., Ltd. (Guangzhou, China). Cells were plated into 6-well plates at a density of $60-70 \%$ confluence. After an overnight incubation, cell transfection was performed using Lipofectamine 2000 Transfection Reagent (Invitrogen; Thermo Fisher Scientific, Inc., Waltham, MA, USA) based on the manufacturer's instructions. Transfected cells were incubated at $37^{\circ} \mathrm{C}$ in a $\mathrm{CO}_{2}$ incubator for $6 \mathrm{~h}$, and culture medium was replaced with fresh RPMI-1640 medium containing $10 \%$ FBS.

RNA isolation and reverse transcription quantitative polymerase chain reaction $(R T-q P C R)$. The total RNA was extracted from the tissues or cells with TRIzol reagent (Invitrogen, Carlsbad, CA, USA). For the miR-382 expression analysis, reverse transcription was performed with TaqMan MicroRNA Reverse Transcription kit (Applied Biosystems, Foster City, CA, USA). Quantitative PCR (qPCR) analyses were conducted with TaqMan MicroRNA PCR kit using the Applied Biosystems ${ }^{\circledR}$ 7900HT Fast Real-Time PCR system (both from Applied Biosystems). To quantify the BDNF mRNA, RNA was reverse transcribed to cDNA from $1 \mu \mathrm{g}$ of total RNA using a PrimeScript RT Reagent kit, followed by qPCR with SYBR Premix Ex Taq ${ }^{\mathrm{TM}}$ kit (both from Takara Bio, Inc., Dalian, China). miR-382 and BDNF mRNA expression levels were normalised to endogenous U6 and glyceraldehyde 3-phosphate dehydrogenase (GAPDH), respectively. Relative expression changes were analysed using the $2^{-\Delta \Delta \mathrm{Cq}}$ method (32).

Cell Counting Kit-8 (CCK-8) assay. CCK-8 assay (Dojindo Molecular Technologies, Inc., Kumamoto, Japan) was used to determine RB cell proliferation. Cells were plated in 96-well plates at a density of $3 \times 10^{3}$ cells per well. After transfection, cells were incubated at $37^{\circ} \mathrm{C}$ in a $5 \% \mathrm{CO}_{2}$ atmosphere for 0,24 , 48 and $72 \mathrm{~h}$. CCK-8 assay was performed at each time point. CCK-8 reagent $(10 \mu \mathrm{l})$ was added into each well, and cells were incubated at $37^{\circ} \mathrm{C}$ for an additional $4 \mathrm{~h}$. Finally, the absorbance was examined at a wavelength of $450 \mathrm{~nm}$ using a multifunction microplate reader (Bio-Rad Laboratories, Inc., Hercules, CA, USA). Each assay was performed in 5-well replication and repeated three times.

Matrigel invasion assay. The invasive capacity of RB cells was examined using 24-well Transwell chambers $(8 \mu \mathrm{m}$; Corning Costar, Cambridge, MA, USA) pre-coated with Matrigel ${ }^{\circledR}$ (BD Biosciences, Franklin Lakes, NJ, USA). After incubation for $48 \mathrm{~h}$, the transfected cells were collected, re-suspended with FBS-free RPMI-1640 medium as a single-cell solution and seeded into the upper chambers at a density of $5 \times 10^{4}$ cells/chamber. RPMI-1640 medium supplemented with $20 \%$ FBS was added into the lower chamber as a chemoattractant. Transwell chambers were then incubated at $37^{\circ} \mathrm{C}$ in a $5 \% \mathrm{CO}_{2}$ atmosphere for $24 \mathrm{~h}$. Subsequently, the non-invasive cells were carefully removed with cotton swab, whereas the invasive cells were fixed with $100 \%$ methanol for $5 \mathrm{~min}$, stained with $0.1 \%$ crystal violet for $10 \mathrm{~min}$ and washed in PBS. The number of invasive cell was counted in five randomly selected fields under an inverted microscope (CKX41; Olympus, Tokyo, Japan).

miR-382 target prediction. The potential targets of miR-382 were analyzed with miRNA target prediction algorithms: PicTar (http://pictar.mdc-berlin.de/) and TargetScan (http://www. targetscan.org/).

Luciferase reporter assay. For luciferase reporter assay, reporter plasmids, pMIRGLO-BDNF-3'-UTR wild-type (Wt) and pMIRGLO-BDNF-3'-UTR mutant (Mut), were synthesised by Shanghai GenePharma Co., Ltd. Cells were seeded into 24 -well plates at a density of $1.5 \times 10^{5}$ per well. After an overnight incubation, cells were transfected with miR-382 mimics or miR-NC, along with pMIRGLO-BDNF-3'-UTR Wt or pMIRGLO-BDNF-3'-UTR Mut, using Lipofectamine 2000 based on the manufacturer's instructions. Cells were harvested $48 \mathrm{~h}$ after transfection, and the luciferase activities were measured using dual-luciferase reporter assay system (Promega, Madison, WI, USA) following the manufacturer's protocol. Renilla luciferase was used for normalisation used in this research. 
Western blot analysis. The total proteins of the cell lines and tissues were extracted using a radioimmunoprecipitation assay buffer (Beyotime, Shanghai, China) in the presence of a proteinase inhibitor cocktail (Sigma, St. Louis, MO, USA). The total protein concentration was examined using the bicinchoninic acid assay protein assay (Pierce Biotechnology, Inc., Rockford, IL, USA). Equal amounts of protein were separated by $10 \%$ sodium dodecyl sulfate-polyacrylamide gel electrophoresis and transferred onto polyvinylidene difluoride membranes (Millipore, Billerica, MA, USA). After blocking with 5\% skim milk in Tris-buffered saline containing $0.05 \%$ Tween-20 (TBST), the membranes were incubated at $4^{\circ} \mathrm{C}$ overnight with primary antibodies: rabbit anti-human polyclonal BDNF (sc-20981; 1:1,000 dilution; Santa Cruz Biotechnology, Santa Cruz, CA, USA), mouse anti-human monoclonal phosphoinositide 3-kinase (PI3K) antibody (ab86714; 1:1,000 dilution; Abcam, Cambridge, MA, USA), rabbit anti-human polyclonal p-PI3K antibody (cat. no. 4228; 1:1,000 dilution; Cell Signaling Technology, Danvers, MA, USA), mouse anti-human monoclonal p-protein kinase B (AKT) (sc-271966; 1:1,000 dilution; Santa Cruz Biotechnology), mouse anti-human monoclonal AKT (sc-56878; 1:1,000 dilution; Santa Cruz Biotechnology), and mouse anti-human monoclonal GAPDH (sc-32233; 1:1,000 dilution; Santa Cruz Biotechnology). The membranes were subsequently washed in TBST thrice and probed with corresponding horseradish peroxidase-conjugated secondary antibody (1:5,000 dilution; Santa Cruz Biotechnology) for $2 \mathrm{~h}$ at room temperature. Finally, the protein signals were detected using Pierce ${ }^{\mathrm{TM}}$ ECL Western Blotting Substrate (Pierce Biotechnology, Inc.) and analysed with Quantity One ${ }^{\circledR}$ software (version 4.62; Bio-Rad Laboratories, Inc.). GAPDH was used as a loading control.

Statistical analysis. All data are expressed as mean \pm standard deviation, and the differences between groups were analysed with Student's t-test or one-way analysis of variance using SPSS version 19.0 software (SPSS, Inc., Chicago, IL, USA). Spearman's correlation analysis was utilised to analyse the correlation between miR-382 and BDNF mRNA expression levels in RB tissues. $\mathrm{P}<0.05$ was considered statistically significant.

\section{Results}

miR-382 expression is downregulated in RB tissues and cell lines. Firstly, we measured miR-382 expression in $26 \mathrm{RB}$ tissues and 9 normal retina tissues using RT-qPCR. The miR-382 expression levels were significantly downregulated in $\mathrm{RB}$ tissues compared with that in normal retina tissues (Fig. 1A, P<0.05). Moreover, the miR-382 expression levels in three RB cell lines (Y79, WERI-RB-1 and SO-RB50) were examined. As shown in Fig. 1B, miR-382 expression decreased in $\mathrm{RB}$ cell lines compared with that in normal retina tissues $(\mathrm{P}<0.05)$. These results suggested that miR-382 may play crucial roles in RB occurrence and development.

miR-382 inhibits $R B$ cell proliferation and invasion in vitro. To determine the potential roles of miR-382 in RB, Y79 and WERI-RB-1 cells were transfected with miR-382 mimics or miR-NC. RT-qPCR confirmed that miR-382 was markedly
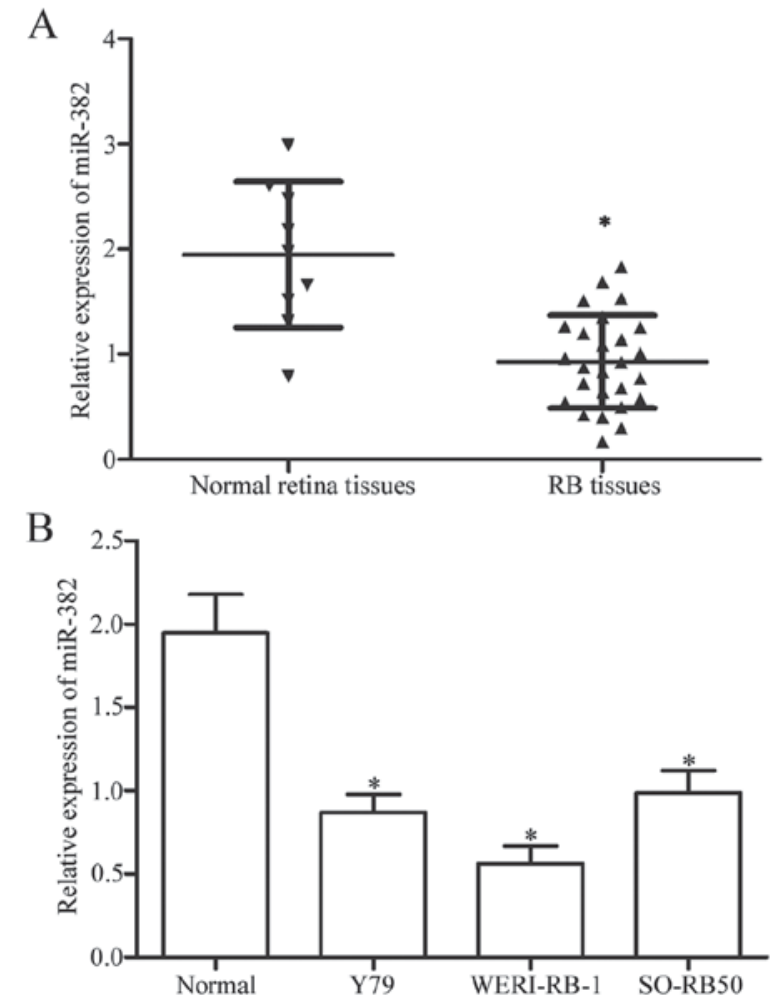

Figure 1. miR-382 was downregulated in RB tissues and cell lines. (A) Expression level of miR-382 was determined in $26 \mathrm{RB}$ tissues and 9 normal retina tissues through $\mathrm{RT}-\mathrm{qPCR}$. (B) miR-382 expression was determined in 3 RB cell lines (Y79, WERI-RB-1 and SO-RB50). ${ }^{*} \mathrm{P}<0.05$ compared with respective control. RB, retinoblastoma; miR-382, microRNA-382.

upregulated in Y79 and WERI-RB-1 cells after transfection with miR-382 mimics ( $\mathrm{P}<0.05$; Fig. $2 \mathrm{~A}$ and $\mathrm{B})$. CCK-8 assay was performed to investigate the effect of miR-382 on RB cell proliferation. The miR-382 upregulation significantly inhibited the Y79 and WERI-RB-1 cell proliferation $(\mathrm{P}<0.05$; Fig. 2C and D). We further performed Matrigel invasion assay to determine the effect of miR-382 on RB cell invasion. The invasive capacities of Y79 and WERI-RB-1 cells were obviously suppressed in cells overexpressing miR-382 compared with those in the miR-NC group ( $\mathrm{P}<0.05$; Fig. $2 \mathrm{E}$ and $\mathrm{F})$. These results suggested that miR-382 may play tumour-suppressing roles in RB.

$B D N F$ is a direct target gene of $m i R-382$ in $R B$. To elucidate the underlying molecular mechanisms of miR-382 in RB, bioinformatics analysis was conducted to predict the putative targets of miR-382. Among these potential targets, BDNF (Fig. 3A) was selected for further validation owing to its potential roles in RB malignancy regulation $(33,34)$. To confirm this prediction, luciferase reporter assay was used to determine whether miR-382 could directly target the 3'-UTR of BDNF. The restoration expression of miR-382 decreased the luciferase activity of reporter plasmid that contained the wide-type BDNF 3'-UTR $(\mathrm{P}<0.05$; Fig. 3B). In contrast, no significant inhibition was observed for the Mut reporter after miR-382 mimic transfection. RT-qPCR and western blot analysis were utilised to detect the BDNF expression in Y79 and WERI-RB-1 cells transfected with miR-382 mimics or miR-NC. As shown in Fig. 3C and D, miR-382 overexpression decreased BDNF expression at both 
A

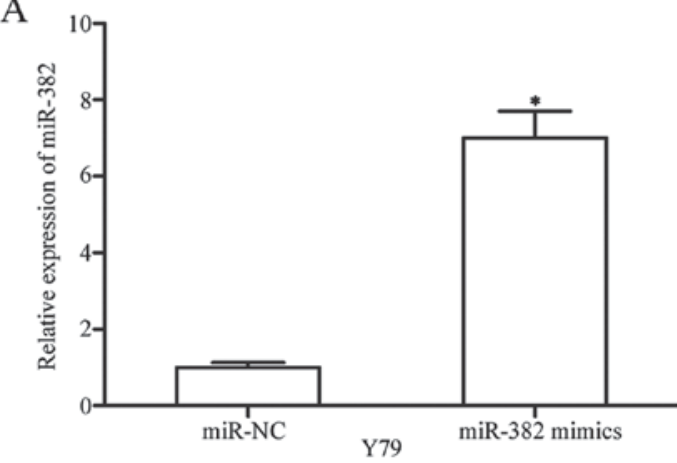

$\mathrm{C}$

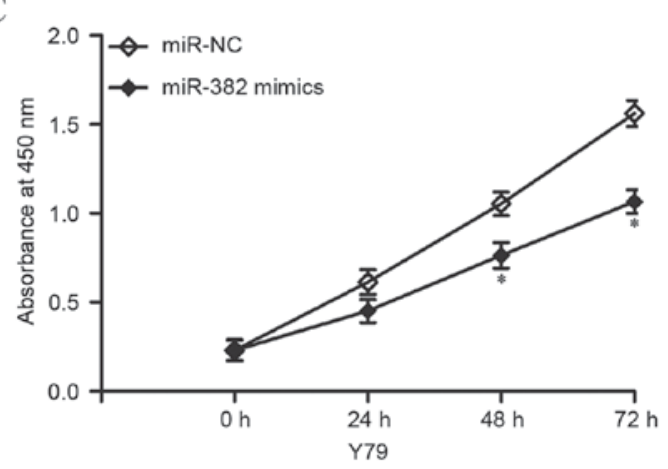

$\mathrm{E}$

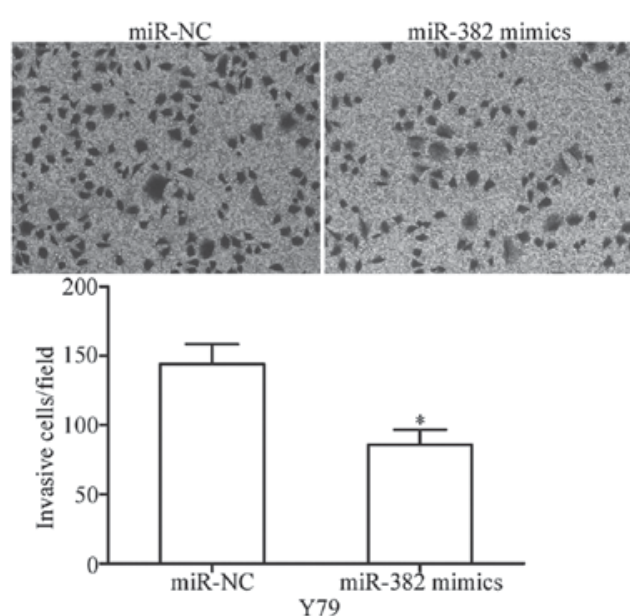

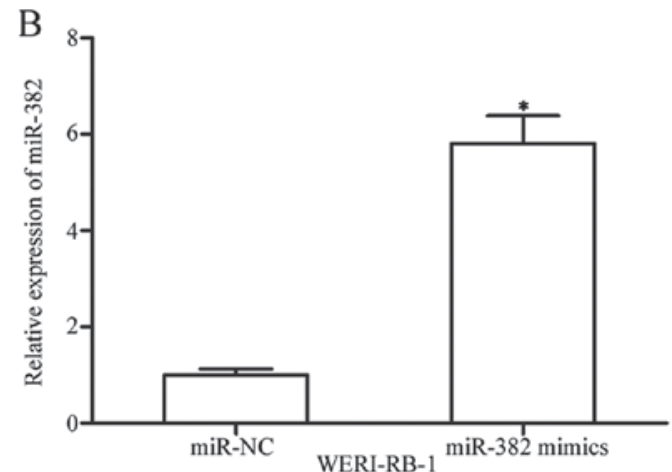

D

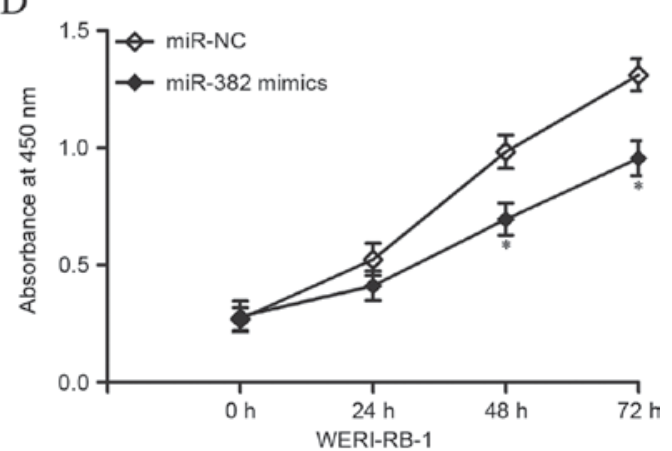

$\mathrm{F}$

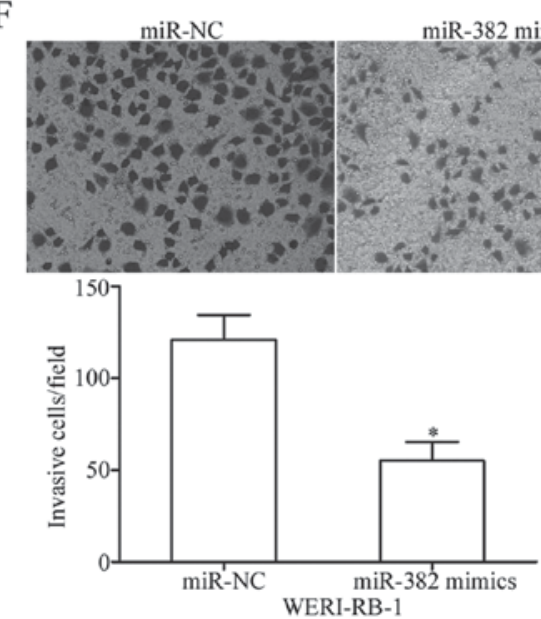

Figure 2. miR-382 overexpression inhibited proliferation and invasion of Y79 and WERI-RB-1 cells. (A and B) The relative miR-382 expression level in Y79 and WERI-RB-1 cells transfected with miR-382 mimics or miR-NC was examined via RT-qPCR. (C and D) CCK-8 assay was performed to evaluate the effect of miR-382 on Y79 and WERI-RB-1 cell proliferation. (E and F) Matrigel invasion assay was used to evaluate the effect of miR-382 on the invasive capacities of Y79 and WERI-RB-1 cells. ${ }^{*} \mathrm{P}<0.05$ compared with respective control. miR-382, microRNA-382; miR-NC, miRNA mimic negative control; RT-qPCR, reverse transcription quantitative polymerase chain reaction; CCK-8, Cell Counting Kit-8.

the mRNA and protein levels in Y79 and WERI-RB-1 cells $(\mathrm{P}<0.05)$. Taken together, these data indicate that BDNF is a direct target of miR-382 in RB.

$B D N F$ is upregulated and inversely correlated with miR-382 expression in $R B$ tissues. To further explore the relationship between miR-382 and BDNF, we detected the BDNF expression at the mRNA and protein levels in $26 \mathrm{RB}$ tissues and nine normal retina tissues using RT-qPCR and western blot analysis, respectively. $\mathrm{RB}$ tissues had increased BDNF expression at both the mRNA $(\mathrm{P}<0.05$; Fig. 4A) and protein levels $(\mathrm{P}<0.05$; Fig. 4B) compared with the normal retina tissues. Additionally, Spearman's correlation analysis indicated a statistically significant inverse correlation between miR-382 and BDNF mRNA level in $\mathrm{RB}$ tissues ( $\mathrm{r}=-0.7284, \mathrm{P}<0.001$; Fig. 4C).
$B D N F$ reverses the tumour-suppressing effects of miR-382 on $R B$ cell proliferation and invasion. Rescue experiments were performed to evaluate whether BDNF is responsible for the tumour-suppressing effects of miR-382 in RB cells. Y79 and WERI-RB-1 cells were transfected with miR-382 mimics or miR-NC and co-transfected with pcDNA3.1-BDNF or pcDNA3.1. Western blot analysis revealed that the restoration expression of BDNF abolished the inhibition caused by the miR-382 mimics in Y79 and WERI-RB-1 cells $(\mathrm{P}<0.05$; Fig. 5A). Subsequently, CCK-8 and Matrigel invasion assays were performed in the above mentioned cells. Notably, the resumption expression of BDNF rescued the suppressive effects of miR-382 on the proliferation $(\mathrm{P}<0.05$; Fig. 5B and $\mathrm{C})$ and invasion $(\mathrm{P}<0.05$; Fig. 5D and $\mathrm{E})$ of $\mathrm{Y} 79$ and WERI-RB-1 cells. These results suggested that miR-382 exerted its 
A

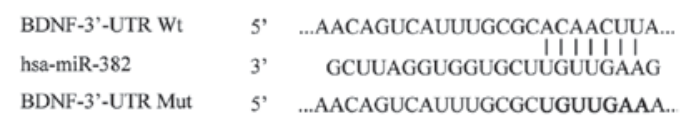

$\mathrm{C}$

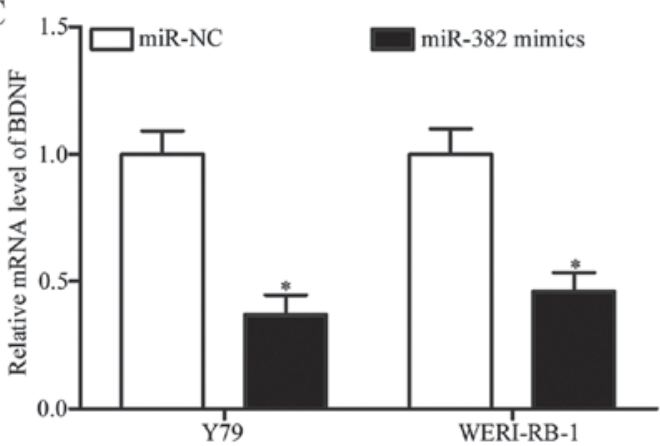

B

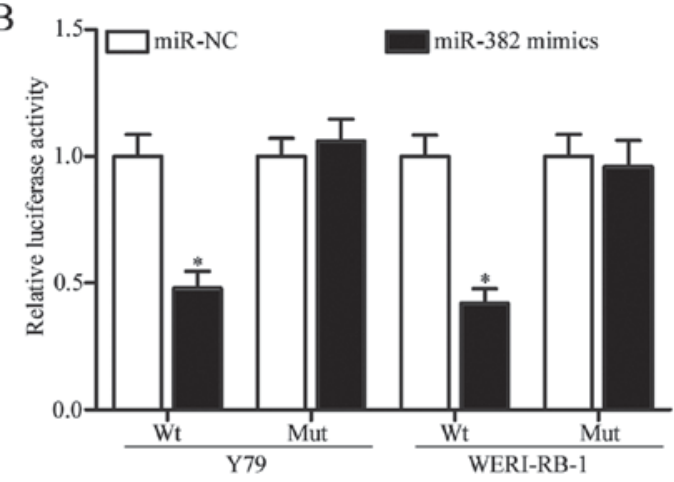

$\mathrm{D}$

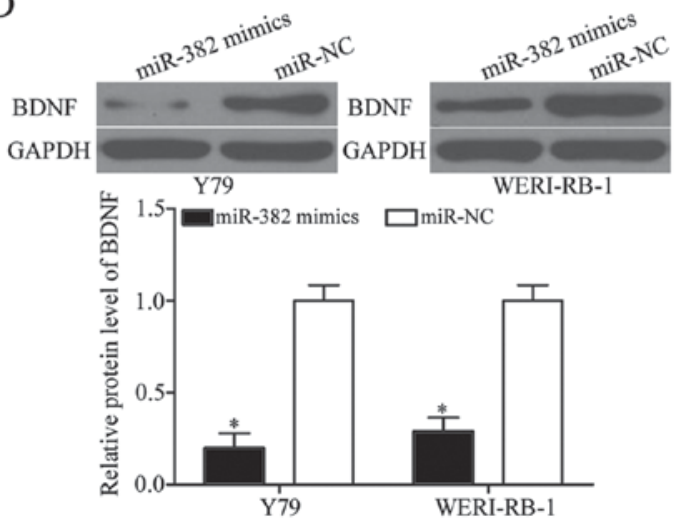

Figure 3. BDNF is the direct target gene of miR-382 in RB. (A) Putative binding site in the 3'-UTR of BDNF. Mutation was generated in the complementary sites for the seed regions in miR-382. (B) Relative luciferase activity was measured in Y79 and WERI-RB-1 cells after transfection with Wt or Mut BDNF reporter plasmid together with miR-382 mimics or miR-NC. (C and D) RT-qPCR and western blot analysis of BDNF expression in Y79 and WERI-RB-1 cells transfected with miR-382 mimics or miR-NC. "P $<0.05$ compared with respective control. BDNF, brain-derived neurotrophic factor; miR-382, microRNA-382; $\mathrm{RB}$, retinoblastoma; 3'-UTR, 3'-untranslated region; Wt, wild-type; Mut, mutant; miR-NC, miRNA mimic negative control; RT-qPCR, reverse transcription quantitative polymerase chain reaction; GAPDH, glyceraldehyde 3-phosphate dehydrogenase.

A

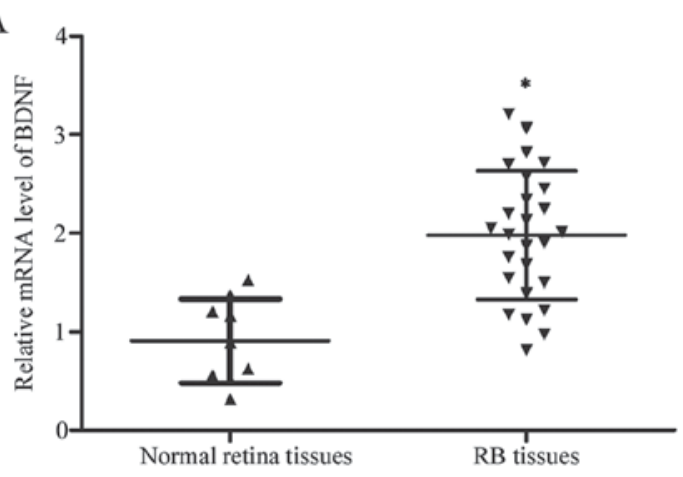

B

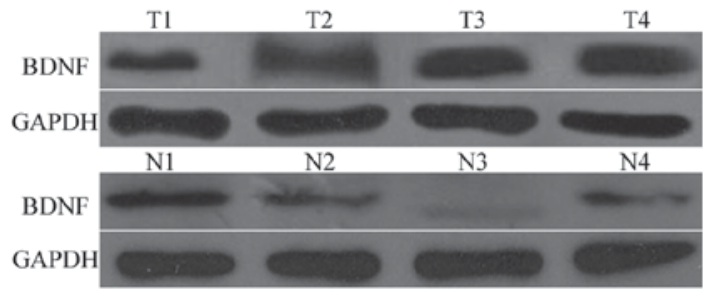

$\mathrm{C}$

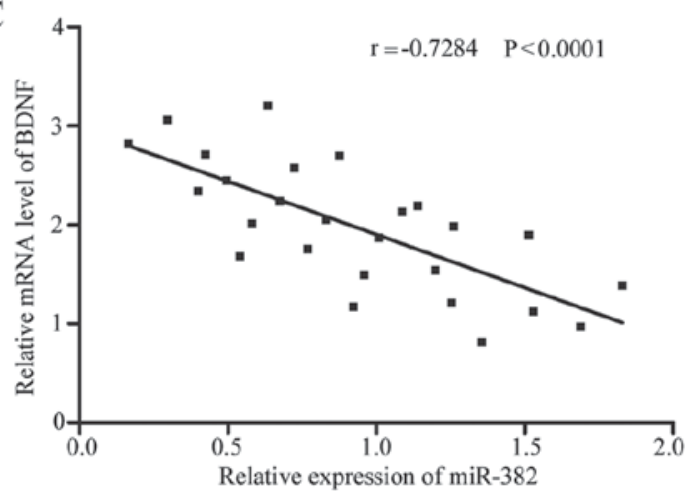

Figure 4. BDNF expression was inversely correlated with miR-382 expression in RB tissues. (A and B) The BDNF mRNA and protein expression levels were determined in RB tissues and normal retina tissues. (C) Negative association between BDNF and miR-382 expression in RB tissues was explored through Spearman's correlation analysis. " $\mathrm{P}<0.05$ compared with respective control. BDNF, brain-derived neurotrophic factor; miR-382, microRNA-382; RB, retinoblastoma; GAPDH, glyceraldehyde 3-phosphate dehydrogenase.

tumour-suppressing roles in $\mathrm{RB}$, at least in part by regulating BDNF.
miR-382 inhibits PI3K/AKT signalling in $R B$. BDNF is involved in PI3K/AKT signalling pathway regulation $(35,36)$. 
A
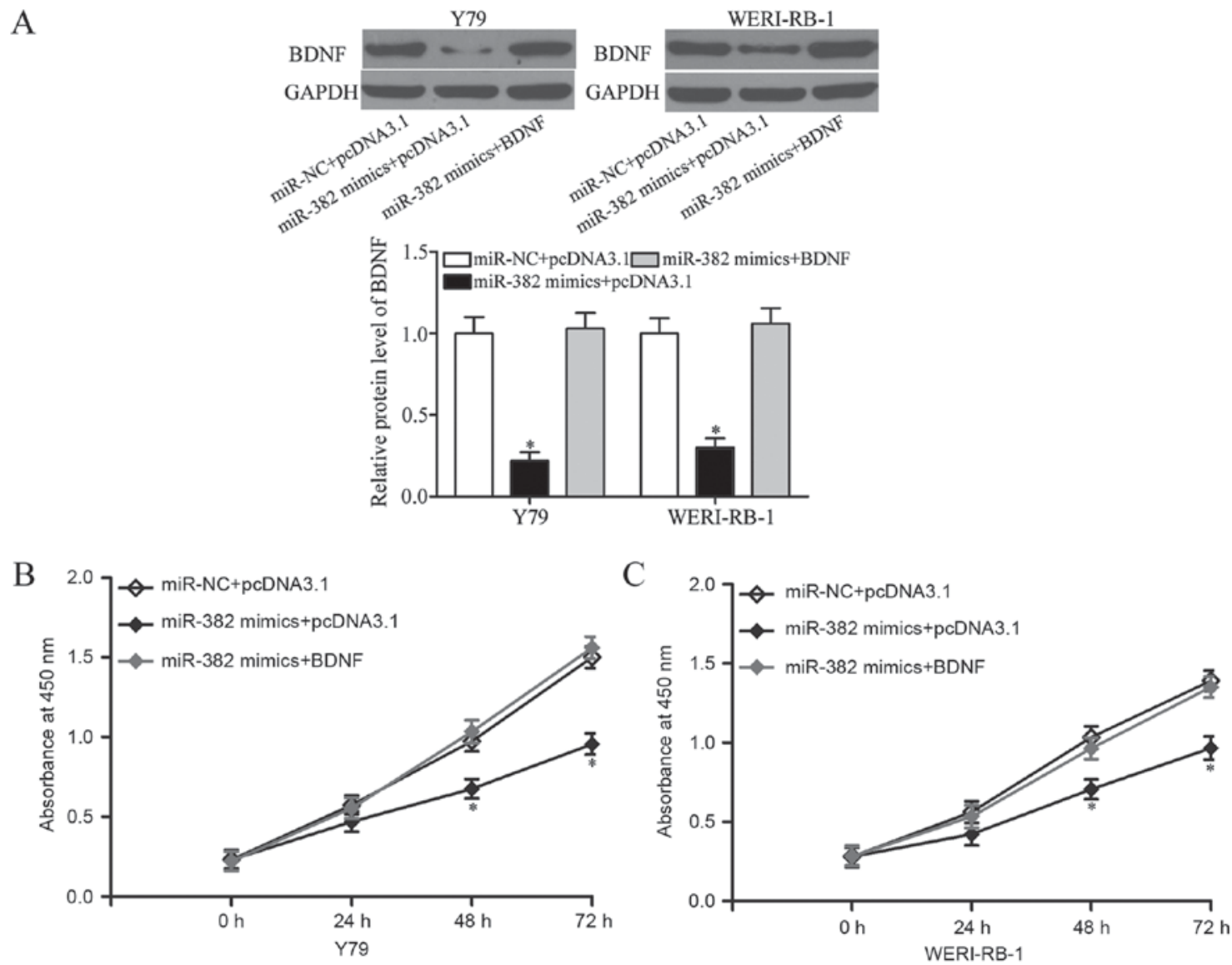

$\mathrm{D}$

$\mathrm{E}$
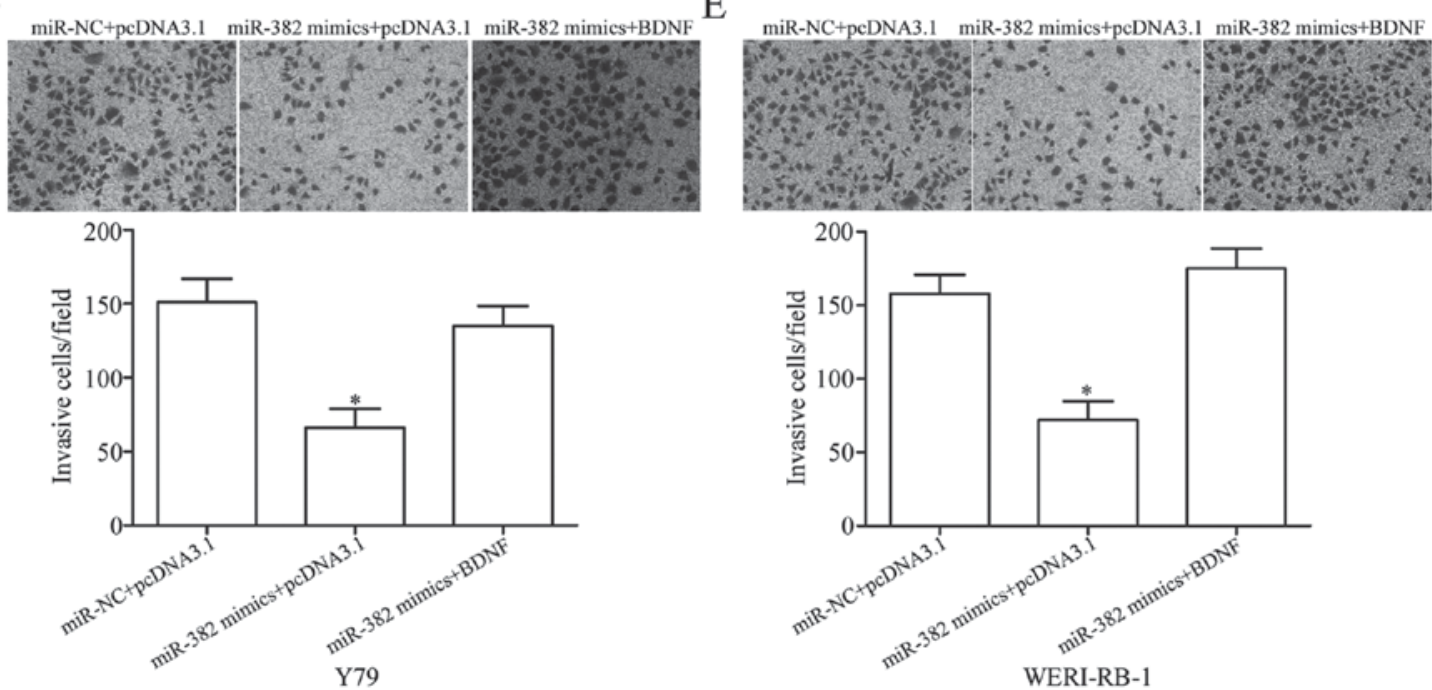

Figure 5. Restoration expression of BDNF reversed the inhibitory effects of miR-382 on the proliferation and invasion of Y79 and WERI-RB-1 cells. (A) BDNF protein was measured through western blot analysis in Y79 and WERI-RB-1 cells transfected with miR-382 mimics with or without pcDNA3.1-BDNF. (B and C) CCK-8 assay was conducted in Y79 and WERI-RB-1 cells transfected with miR-382 mimics with or without pcDNA3.1-BDNF. (D and E) Matrigel invasion assay was performed in Y79 and WERI-RB-1 cells transfected with miR-382 mimics with or without pcDNA3.1-BDNF. "P<0.05 compared with respective control. BDNF, brain-derived neurotrophic factor; miR-382, microRNA-382; CCK-8, Cell Counting Kit-8; GAPDH, glyceraldehyde 3-phosphate dehydrogenase; miR-NC, miRNA mimic negative control.

Therefore, we analysed the effects of miR-382 on PI3K, p-PI3K, AKT and p-AKT protein expression in Y79 and WERI-RB-1 cells transfected with miR-382 mimics or miR-NC. The miR-382 upregulation was found to reduce the p-PI3K and p-AKT expression but did not affect the total PI3K and AKT expression in Y79 and WERI-RB-1 cells (Fig. 6). These results suggested that miR-382 exerts suppressive roles in RB cells by directly regulating BDNF and indirectly regulating the PI3K/AKT signalling pathway.

\section{Discussion}

miRNAs serve as post-transcriptional gene regulators that potentially play critical roles in the proliferation, cycle, 


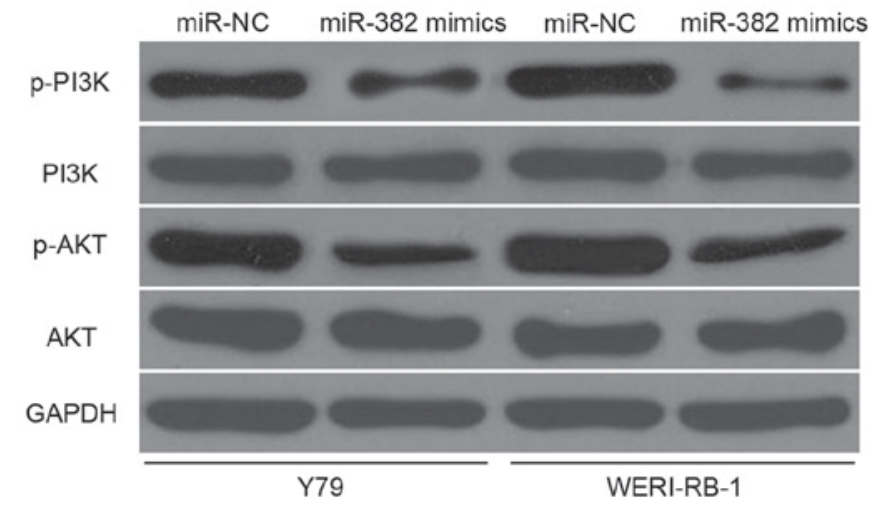

Figure 6. miR-382 inhibits PI3K/AKT signalling in Y79 and WERI-RB-1 cells. Western blot analysis was carried out to detect the expression levels of PI3K, p-PI3K, AKT and p-AKT in Y79 and WERI-RB-1 cells transfected with miR-382 mimics or miR-NC. miR-382, microRNA-382; PI3K, phosphoinositide 3-kinase; AKT, protein kinase B; miR-NC, miRNA mimic negative control; GAPDH, glyceraldehyde 3-phosphate dehydrogenase.

differentiation, angiogenesis and metastasis of various kinds of cancer cells (37-39). Multiple miRNAs are aberrantly expressed in $\mathrm{RB}$ and contribute to RB initiation and progression $(22,40,41)$. However, the expression and roles of miR-382 in $\mathrm{RB}$ remain unclear. In this study, miR-382 was significantly downregulated in RB tissues and cell lines. Furthermore, miR-382 upregulation suppressed cell proliferation and invasion of RB in vitro. BDNF was also demonstrated to be a direct target of miR-382 in RB. Importantly, miR-382 inactivated PI3K/AKT signalling pathway in RB. To the best of our knowledge, this is the first study to investigate the expression and role of miR-382 in RB.

miR-382 was frequently found to be abnormally expressed in multiple human cancers. For instance, miR-382 was significantly downregulated in non-small cell lung cancer. Additionally, low miR-382 expression level was correlated with the tumour stage and metastasis of non-small cell lung cancer (29). The miR-382 expression level was decreased in highly metastatic osteosarcoma cell lines and relapsed osteosarcoma tissues. Low miR-382 expression level was inversely associated with relapse and positively associated with metastasis-free survival in osteosarcoma patients (42). The miR-382 expression was relatively low in oesophageal squamous cell carcinoma for patients with poor outcome. Kaplan-Meier analysis indicated a significant reverse correlation between miR-382 expression and survival time of oesophageal squamous cell carcinoma patients (43). The downregulation of miR-382 was also observed in prostate (44), ovarian (45) and colorectal (30) cancers. However, miR-382 was highly expressed in tumour tissues and associated with pathological grade and clinical stage in breast cancer (31). These findings indicated that miR-382 expression has tissue specificity and may be a prognostic target for cancers.

Aberrant miR-382 expression was reportedly associated with various human cancers. Chen et al found that ectopic miR-382 expression repressed cell proliferation, migration and invasion in non-small cell lung cancer (29). Xu et al revealed that miR-382 overexpression attenuated osteosarcoma cell growth, metastasis, epithelial-mesenchymal transition and chemoresistance $(42,46)$. Zhang et al reported that restoration expression of miR-382 impeded cell proliferation and motility in prostate cancer (44). Tan et al demonstrated that resumption expression of miR-382 decreased cell proliferation, metastasis and epithelial-mesenchymal transition in ovarian cancer (45). Zhou et al revealed that enforced miR-382 expression inhibited colorectal cancer cell growth, migration and invasion in vitro (30). These studies indicate that miR-382 is a tumour suppressor in certain types of cancer. However, miR-382 serve as an oncogene in breast cancer by promoting cell viability, clonogenicity, survival, migration and invasion in vitro and tumourigenesis or metastasis in vivo (31). This contradiction may be explained by the 'imperfect complementarity' of the interactions between miRNAs and their target genes (47).

Several targets of miR-382 have been identified, such as SETD8 (29) in lung cancer, KLF12 (46), HIPK3 (46), YB-1 (42) in osteosarcoma, COUP-TFII (44) in prostate cancer, ROR1 (45) in ovarian cancer, NR2F2 in colorectal cancer (30), and RERG (31) in breast cancer. In this study, BDNF was demonstrated to be a direct downstream target gene of miR-382 in RB. To explore the mechanism underlying the tumor-suppressing roles of miR-382 in $\mathrm{RB}$, bioinformatics analysis was performed to predict the candidate targets of miR-382. BDNF was predicted as a potential target of miR-382. Luciferase reporter assay was conducted to confirm this prediction, and found that miR-382 could directly targeted the 3'-UTR of BDNF. Additionally, RT-qPCR and western blot analysis demonstrated that miR-382 negatively regulated endogenous BDNF expression at both mRNA and protein level in RB cells. Furthermore, BDNF was upregulated in RB tissues and inverse correlated with miR-382 expression level. Besides, rescue experiments indicated that BDNF overexpression reversed the suppressive effects of miR-382 on RB cells. Taken together, it is reasonable to suggest that alterations in miR-382 expression regulates the proliferation and invasion of $\mathrm{RB}$ cells via directly targeting BDNF.

BDNF, a member of the neurotrophin family, plays an essential role in neural maturation and differentiation (48). BDNF was upregulated in several human malignancies, such as bladder cancer (49), glioma, gastric cancer (50), colorectal cancer (51) and breast cancer (52). Moreover, aberrant BDNF expression was involved in tumourigenesis and tumour development. In colorectal cancer, BDNF knockdown inhibited cell proliferation, migration and invasion and enhanced apoptosis $(51,53)$. In lung cancer, BDNF downregulation suppressed cell proliferation and invasion and increased apoptosis (54). In $\mathrm{RB}, \mathrm{BDNF}$ was upregulated in tumour tissues and cell lines (33) and contribute in RB occurrence and progression regulation (34). All these findings suggested that BDNF is worthy of investigation as a potential target for the treatment of patients with RB.

In conclusion, miR-382 was significantly downregulated in RB tissues and cells. Additionally, we demonstrated that miR-382 acts as a tumour suppressor in RB through direct targeting of BDNF and indirect regulation of the PI3K/AKT signalling pathway. These results contribute evidence that miR-382 plays an important role in tumour malignant progress, and miR-382 may represent a potential gene-targeting approach for RB treatments. 


\section{References}

1. Abramson DH, Shields CL, Munier FL and Chantada GL: Treatment of retinoblastoma in 2015: Agreement and disagreement. JAMA Ophthalmol 133: 1341-1347, 2015.

2. Broaddus E, Topham A and Singh AD: Incidence of retinoblastoma in the USA: 1975-2004. Br J Ophthalmol 93: 21-23, 2009.

3. Kleinerman RA, Schonfeld SJ and Tucker MA: Sarcomas in hereditary retinoblastoma. Clin Sarcoma Res 2: 15, 2012.

4. Marees T, Moll AC, Imhof SM, de Boer MR, Ringens PJ and van Leeuwen FE: Risk of second malignancies in survivors of retinoblastoma: More than 40 years of follow-up. J Natl Cancer Inst 100: 1771-1779, 2008.

5. Moll AC, Imhof SM, Bouter LM and Tan KE: Second primary tumors in patients with retinoblastoma. A review of the literature. Ophthalmic Genet 18: 27-34, 1997.

6. Benavente CA and Dyer MA: Genetics and epigenetics of human retinoblastoma. Annu Rev Pathol 10: 547-562, 2015.

7. Yang YQ, Li J and Yuan HF: Epidemiology and risk factors of retinoblastoma in Chongqing area. Int J Ophthalmol 9: 984-988, 2016.

8. Finger PT, Harbour JW and Karcioglu ZA: Risk factors for metastasis in retinoblastoma. Surv Ophthalmol 47: 1-16, 2002.

9. Friedman DL, Himelstein B, Shields CL, Shields JA, Needle M, Miller D, Bunin GR and Meadows AT: Chemoreduction and local ophthalmic therapy for intraocular retinoblastoma. J Clin Oncol 18: 12-17, 2000.

10. Dimaras H, Kimani K, Dimba EA, Gronsdahl P, White A Chan HS and Gallie BL: Retinoblastoma. Lancet 379: 1436-1446, 2012.

11. Kaliki S, Shields CL, Rojanaporn D, Al-Dahmash S, McLaughlin JP, Shields JA and Eagle RC Jr: High-risk retinoblastoma based on international classification of retinoblastoma: Analysis of 519 enucleated eyes. Ophthalmology 120: 997-1003, 2013.

12. Ha M and Kim VN: Regulation of microRNA biogenesis. Nat Rev Mol Cell Biol 15: 509-524, 2014.

13. Kozomara A and Griffiths-Jones S: miRBase: Integrating microRNA annotation and deep-sequencing data. Nucleic Acids Res 39 (Database Issue): D152-D157, 2011.

14. Bartel DP: MicroRNAs: Genomics, biogenesis, mechanism, and function. Cell 116: 281-297, 2004.

15. Baranwal S and Alahari SK: miRNA control of tumor cell invasion and metastasis. Int J Cancer 126: 1283-1290, 2010

16. Iorio MV and Croce CM: MicroRNA dysregulation in cancer: Diagnostics, monitoring and therapeutics. A comprehensive review. EMBO Mol Med 4: 143-159, 2012.

17. Calin GA and Croce CM: MicroRNA signatures in human cancers. Nat Rev Cancer 6: 857-866, 2006.

18. Gao HY, Huo FC, Wang HY and Pei DS: MicroRNA-9 inhibits the gastric cancer cell proliferation by targeting TNFAIP8. Cell Prolif: 50, 2017.

19. Li C, Lu S and Shi Y: MicroRNA-187 promotes growth and metastasis of gastric cancer by inhibiting FOXA2. Oncol Rep 37: $1747-1755,2017$

20. Zeng Y, Zhu J, Shen D, Qin H, Lei Z, Li W, Liu Z and Huang JA: MicroRNA-205 targets SMAD4 in non-small cell lung cancer and promotes lung cancer cell growth in vitro and in vivo. Oncotarget 8: 30817-30829, 2017.

21. Dong F, Xu T, Shen Y, Zhong S, Chen S, Ding Q and Shen Z: Dysregulation of miRNAs in bladder cancer: Altered expression with aberrant biogenesis procedure. Oncotarget 8: 27547-27568, 2017.

22. Wang LL, Hu HF and Feng YQ: Suppressive effect of microRNA-143 in retinoblastoma. Int J Ophthalmol 9: 1584-1590, 2016.

23. Hwang HW and Mendell JT: MicroRNAs in cell proliferation, cell death, and tumorigenesis. Br J Cancer 96 (Suppl): R40-R44, 2007.

24. Han RL, Wang FP, Zhang PA, Zhou XY and Li Y: miR-383 inhibits ovarian cancer cell proliferation, invasion and aerobic glycolysis by targeting LDHA. Neoplasma 64: 244-252, 2017.

25. Li B, Xie Z, Li Z, Chen S and Li B: MicroRNA-613 targets FMNL2 and suppresses progression of colorectal cancer. Am J Transl Res 8: 5475-5484,2016.

26. Sun Z, Zhang A, Jiang T, Du Z, Che C and Wang F: MiR-145 suppressed human retinoblastoma cell proliferation and invasion by targeting ADAM19. Int J Clin Exp Pathol 8: 14521-14527, 2015.

27. Montoya V, Fan H, Bryar PJ, Weinstein JL, Mets MB, Feng G Martin J, Martin A, Jiang H and Laurie NA: Novel miRNA-31 and miRNA-200a-mediated regulation of retinoblastoma proliferation. PLoS One 10: e0138366, 2015.
28. Shen F, Mo MH, Chen L, An S, Tan X, Fu Y, Rezaei K, Wang Z, Zhang L and Fu SW: MicroRNA-21 down-regulates Rb1 expression by targeting PDCD4 in retinoblastoma. J Cancer 5: 804-812, 2014.

29. Chen T, Ren H, Thakur A, Yang T, Li Y, Zhang S, Wang T and Chen M: miR-382 inhibits tumor progression by targeting SETD8 in non-small cell lung cancer. Biomed Pharmacother 86: 248-253, 2017.

30. Zhou B, Song J, Han T, Huang M, Jiang H, Qiao H, Shi J and Wang Y: MiR-382 inhibits cell growth and invasion by targeting NR2F2 in colorectal cancer. Mol Carcinog 55: 2260-2267, 2016.

31. Ho JY, Hsu RJ, Liu JM, Chen SC, Liao GS, Gao HW and Yu CP: MicroRNA-382-5p aggravates breast cancer progression by regulating the RERG/Ras/ERK signaling axis. Oncotarget 8 : 22443-22459, 2017.

32. Livak KJ and Schmittgen TD: Analysis of relative gene expression data using real-time quantitative PCR and the 2(-Delta Delta C(T)) method. Methods 25: 402-408, 2001.

33. Stephan H, Zakrzewski JL, Bölöni R, Grasemann C, Lohmann DR and Eggert A: Neurotrophin receptor expression in human primary retinoblastomas and retinoblastoma cell lines. Pediatr Blood Cancer 50: 218-222, 2008

34. Shang W, Yang Y, Zhang J and Wu Q: Long noncoding RNA BDNF-AS is a potential biomarker and regulates cancer development in human retinoblastoma. Biochem Biophys Res Commun: Jan 26, 2017 (Epub ahead of print).

35. Mao XY, Zhou HH, Li X and Liu ZQ: Huperzine A alleviates oxidative glutamate toxicity in hippocampal HT22 cells via activating BDNF/TrkB-dependent PI3K/Akt/mTOR signaling pathway. Cell Mol Neurobiol 36: 915-925, 2016.

36. Xia H, Li Y and Lv X: MicroRNA-107 inhibits tumor growth and metastasis by targeting the BDNF-mediated PI3K/AKT pathway in human non-small lung cancer. Int J Oncol 49: 1325-1333, 2016.

37. Huang Q, Liu L, Liu CH, You H, Shao F, Xie F, Lin XS, Hu SY and Zhang $\mathrm{CH}$ : MicroRNA-21 regulates the invasion and metastasis in cholangiocarcinoma and may be a potential biomarker for cancer prognosis. Asian Pac J Cancer Prev 14: 829-834, 2013.

38. Yu SH, Zhang CL, Dong FS and Zhang YM: miR-99a suppresses the metastasis of human non-small cell lung cancer cells by targeting AKT1 signaling pathway. J Cell Biochem 116: 268-276, 2015.

39. Funamizu N, Lacy CR, Parpart ST, Takai A, Hiyoshi Y and Yanaga K: MicroRNA-301b promotes cell invasiveness through targeting TP63 in pancreatic carcinoma cells. Int J Oncol 44: 725-734, 2014.

40. Wu X, Zeng Y, Wu S, Zhong J, Wang Y and Xu J: MiR-204, down-regulated in retinoblastoma, regulates proliferation and invasion of human retinoblastoma cells by targeting CyclinD2 and MMP-9. FEBS Lett 589: 645-650, 2015.

41. Wang J, Wang X, Wu G, Hou D and Hu Q: MiR-365b-3p, down-regulated in retinoblastoma, regulates cell cycle progression and apoptosis of human retinoblastoma cells by targeting PAX6. FEBS Lett 587: 1779-1786, 2013.

42. Xu M, Jin H, Xu CX, Sun B, Song ZG, Bi WZ and Wang Y: miR-382 inhibits osteosarcoma metastasis and relapse by targeting Y box-binding protein 1. Mol Ther 23: 89-98, 2015.

43. Qi B, Lu JG, Yao WJ, Chang TM, Qin XG, Ji YH, Wang TY, Liu SG, Li HC, Liu YZ and Zhao BS: Downregulation of microRNA-382 is associated with poor outcome of esophageal squamous cell carcinoma. World J Gastroenterol 21: 6884-6891, 2015

44. Zhang W, Liu J, Qiu J, Fu X, Tang Q, Yang F, Zhao Z and Wang H: MicroRNA-382 inhibits prostate cancer cell proliferation and metastasis through targeting COUP-TFII. Oncol Rep 36: $3707-3715,2016$

45. Tan H, He Q, Gong G, Wang Y, Li J, Wang J, Zhu D and Wu X miR-382 inhibits migration and invasion by targeting ROR1 through regulating EMT in ovarian cancer. Int J Oncol 48: 181-190, 2016.

46. Xu M, Jin $\mathrm{H}, \mathrm{Xu} \mathrm{CX}$, Sun $\mathrm{B}$, Mao Z, Bi WZ and Wang $\mathrm{Y}$ : miR-382 inhibits tumor growth and enhance chemosensitivity in osteosarcoma. Oncotarget 5: 9472-9483, 2014.

47. Yu Z, Ni L, Chen D, Zhang Q, Su Z, Wang Y, Yu W, Wu X, Ye J, Yang $\mathrm{S}$, et al: Identification of miR-7 as an oncogene in renal cell carcinoma. J Mol Histol 44: 669-677, 2013.

48. McAllister AK: Neurotrophins and neuronal differentiation in the central nervous system. Cell Mol Life Sci 58: 1054-1060, 2001.

49. Lai PC, Chiu TH and Huang YT: Overexpression of BDNF and TrkB in human bladder cancer specimens. Oncol Rep 24: $1265-1270,2010$ 
50. Choi B, Lee EJ, Shin MK, Park YS, Ryu MH, Kim SM, Kim EY, Lee HK and Chang EJ: Upregulation of brain-derived neurotrophic factor in advanced gastric cancer contributes to bone metastatic osteolysis by inducing long pentraxin 3 . Oncotarget 7: 55506-55517, 2016.

51. Yang X, Martin TA and Jiang WG: Biological influence of brain-derived neurotrophic factor (BDNF) on colon cancer cells. Exp Ther Med 6: 1475-1481, 2013

52. Yang X, Martin TA and Jiang WG: Biological influence of brain-derived neurotrophic factor on breast cancer cells. Int J Oncol 41: 1541-1546, 2012.
53. Huang SM, Lin C, Lin HY, Chiu CM, Fang CW, Liao KF, Chen DR and Yeh WL: Brain-derived neurotrophic factor regulates cell motility in human colon cancer. Endocr Relat Cancer 22: 455-464, 2015.

54. Zhang SY, Hui LP, Li CY, Gao J, Cui ZS and Qiu XS: More expression of BDNF associates with lung squamous cell carcinoma and is critical to the proliferation and invasion of lung cancer cells. BMC Cancer 16: 171, 2016. 